\title{
Goethes Anteil an der Lehre von der Aphasie.
}

\author{
Von \\ Dr. Erich Ebstein, \\ Oberarzt an der med. Klinik in Leipzig. \\ (Eingegangen am 12. April 1913.)
}

Die Geschichte der Aphasie ist noch nicht geschrieben worden, obschon Ansätze dazu vorhanden sind ${ }^{1}$ ).

Der Ausdruck „Aphasie“ stammt von einem geborenen Griechen und ausgezeichneten Hellenisten Crisaphis, wie Trousseau ${ }^{2}$ ) erzählt.

Deshalb verwerfen sowohl Broca ${ }^{3}$ ), der 1861 den Ausdruck „,Aphemie" prägte, wie Troussea u diesen Ausdruck durchaus.

Im Jahre 1820 wurde das, was wir heute Aphasie nennen, von Lordat4) mit dem Namen "Alalie“ belegt. Lordat hatte selbst das Unglück, 1828 aphatisch zu werden; er schrieb diese Störung aber nicht einer bestimmten Stelle des Gehirns zu, sondern vielmehr einer Zerrüttung der Synergie der Muskeln, die beim Sprechen beteiligt sind.

Inzwischen hatte Franz Josef Gall $\left.{ }^{5}\right)(1758-1827)$ die Vermutung ausgesprochen, daß die Fähigkeit zum Reden in den vorderen Gehirnlappen ihren Sitz habe, und zwar in der untersten Windung des Stirnlappens, und Bouilla ud ${ }^{6}$ ), ein Schüler Galls, sagte schon 1825, daß das Organ der artikulierten Rede seinen Sitz im vorderen Teile des Gehirns habe. In den „Phrenological Transactions", Vol. III, veröffentlichte Thomas $\operatorname{Hood}^{7}$ ) den ersten durch Autopsie illustrierten Fall von Aphasie aus dem Jahre 1822, also drei Jahre vor Bouillaud.

1) Kußmaul, Die Störungen der Sprache. 3. Aufl. 1885, und Jastrowitz, Historische Notiz über Aphasie. Berl. klin. Wochenschrift 1875, Nr. 23.

2) Trousseau, Medicinische Klinik usw. Bd. 2. Würzburg 1868, S. 612

3) P. Broca, Sur le siège du langage articulé, avec deux observations d'aphémie. Bull. de la soc. anatomique. Bd. 4. 1861.

$\left.{ }^{4}\right)$ Lordat, Rev. per. de la soc. de méd. de Paris. Déc. 1820, p. 317.

$\left.{ }^{5}\right)$ Gall, Anatomie et physiologie du système nerveux. 4 Bde. mit Atlas 1810-1818. - Liepmann, Deutsche med. Wochenschr. 1909, Nr. 22, und A. Frorie p, Die Lehren Fr. J. Galls. Lpz. 1911.

$\left.{ }^{6}\right)$ Bouillaud, Traité de l'encéphalite. 1825, p. 285.

7) Zitiert nach Möbi us, Goethe, Gall, Lpz. 1905, S. 114, und J. Loe b, Einleitung in die vergleichende Gehirnphysiologie. Lpz. 1890, S. 169. 
Im Jahre 1836 brachte Marcus $\mathrm{Dax}^{1}$ ) vor dem Congrès méridional von Montpellier die Mitteilung eines eigenen Sprachzentrums; er fand Unfähigkeit zu sprechen bei Erkrankung der linken Gehirnhälfte.

Bereits 1848 konnte Bouillaud2) 700 Sektionsbefunde über die Bedeutung des Sprachzentrums vorlegen.

Nach Otto Bryk ${ }^{3}$ ) muß, als wirklich erster Entdecker des Sprachzentrums übrigens Goethe gelten. Er hat die Bestimmtheit der hieran geknüpften Ausfallserscheinung zuerst, wenn auch nicht in ausgesprochener Absicht, beschrieben". Bryk verweist dabei in der dazugehörigen Anmerkung auf Goethes Wilhelm Meister, Lehrjahre. 7. Buch, 6. Kapitel - und beruft sich bei diesem Fund auf eine mündliche Mitteilung des Herrn Dr. Otto Pötzl, festgestellt durch Herrn A. Bonvicini, Wien, fügt allerdings hinzu: „Doch bedarf es wohl keiner näheren Ausführung, um zu zeigen, daß alle Vorläufer Brocas (Goethe, Gall, Bouillaud, Dax) von der klinischen Auffassung im heutigen Sinne weit entfernt waren."

Dagegen erwähnt H. Vierordt ${ }^{4}$ ) „,mehr der Kuriosität halber", daß Goethe deutlich eine rechtsseitige Lähmung mit motorischer Aphasie (Unmöglichkeit der Aussprache des richtig vorgestellten Wortes) beschreibt.

In Goethes Wilhelm Meisters Lehrjahren ${ }^{5}$ ) - 7. Band, 6. Kapitel findet sich nun in der ,Geschichte eines deutschen Mädchens", die Therese erzählt, diese Stelle, die in dem Rahmen, wie ich weiter unten zeigen werde, volle Beachtung verdient, weil sie Goethe als einen ausgezeichneten Beobachter und Beschreiber von Krankheitszuständen zeigt, der von der Medizin bekanntlich sagte: „,sie beschäftigt den ganzen Menschen, weil sie sich mit dem ganzen Menschen beschäftigt." Der Kranke, um den es sich handelt, ist Theresens Vater; es heißt dort:

„Wir waren nun frei und lebten wie im Himmel ; . . . Aber leider dauerte dieser frohe Zustand nicht lange, ganz unvermutet ward mein Vater von einem Schlag. flusse befallen, der ihm die rechte Seite lähmte und den reinen Gebrauch der Sprache benahm. Man mußte alles erraten, was er verlangte; denn er brachte nie das Wort hervor, das er im Sinne hatte. Sehr ängstlich waren wir daher manche Augenblicke, in denen er mit mir ausdrücklich allein sein wollte; er deutete mit heftiger Gebärde, daß jedermann sich entfernen sollte, und wenn wir uns allein sahen, war er nicht

1) Marcus Dax, zitiert nach Trousseau, Medizinische Klinik, Bd. 2, Würzburg 1868, S. $636 \mathrm{f}$.

2) Bouillaud, Bull. de I'Acad. de Médecine. XIII, p. 699.

3) Otto Bryk, Entwicklungsgeschichte der . . Naturwissenschaft . . . Lpz. 1909, I. Bd., S. 560 .

4) H. Vierordt, Medizinisches aus der Geschichte. 3. Auflage 1910, S. 118.

5) Goethe, hg. von Maync. Bd. 10. Bibliograph. Institut. S, 32 u. S. 465 f. Die erste Ausgabe in 4 Bänden. Berlin, bei J. F. Unger, 1795-1796, und Weimarer Sophien-Ausgabe Bd. 23. (1901), S. 50. 
imstande, das rechte Wort hervorzubringen. Seine Ungeduld stieg aufs äußerste, und sein Zustand betrübte mich im innersten Herzen. So viel schien mir gewiß, daß er mir etwas zu vertrauen hatte, das mich besonders anging. Welches Verlangen fühlt ich nicht, es zu erfahren! Sonst konnt ich ihm alles an den Augen ansehen; aber jetzt war es vergebens! Selbst seine Augen sprachen nicht mehr. Nur so viel war mir deutlich: er wollte nichts, er begehrte nichts, er strebte nur, mir etwas zu entdecken, das ich leider nicht erfuhr. Sein Übel wiederholte sich, er ward bald darauf ganz untätig und unfähig und nicht lange, so war er tot".

Aus dieser Stelle des 7. Buches in Goethes Wilhelm Meister, das schon im Mai 1796 im Manuskript Schiller vorlag, entnehme ich nicht, wie Bryk es tut, daß Goethe der erste Entdecker des Sprachzentrums gewesen ist, sondern daß er offenbar auf Grund eines von ihm im Leben beobachteten Falles von motorischer Aphasie ${ }^{1}$ ) diesen Symptomenkomplex klinisch richtig beobachtete und kurz, aber treffend beschrieben hat. Wer das Vorbild des geschilderten Kranken gewesen ist, darüber schweigen sich alle Erklärer des Wilhelm Meister aus; denn Goethe hüllte gern die ihm als Unterlage zu seinen Gestalten dienenden Vorbilder berechtigt in ein gewisses Dunkel, so daß Schiller am 20. Oktober 1797 dieses Werk Goethes, - wohl auch in diesem Sinne, - ,inkalkulabelste Produktion" nennen konnte.

Nun ersehe ich zwar aus einer Notiz bei H. Vierordt (l. c.) — während ich dieses niederschreibe - , daß Goethes mütterlicher Großvater Textor in dieser Art d. h. aphatisch vom rechtsseitigen Schlaganfall getroffen und drei Jahre später daran gestorben sei. Auch Möbius, (Goethe, Teil I, S. 234 Anm.) meint, daß Goethe vielleicht an Textor dachte, als er den Fall schilderte. Wie ich diese Sache nun weiter verfolge, finde ich bei H.Düntzer in dem Buche ,Goethes Stammbäume“" (Gotha 1894) nun tatsächlich die Bemerkung, daß, als Goethe am 3. September 1768 aus Leipzig nach Frankfurt krank ${ }^{2}$ ) zurückkehrte, der Großvater Johann Wolfgang Textor (geb. 1693, gest. 6. Februar 1771) ,ohne Hoffnung völliger Herstellung darniederlag. Ein Schlagfluß hatte den rastlos tätigen ... in seiner Amtsstube befallen, ihm den rechten Arm und die Zunge gelähmt, auch seinen Geist angegriffen." Als Goethe im April 1770 nach Straßburg ging, hatte er den Großvater sehr leidend verlassen. Als der Schultheiß Textor am 6. Februar 1771 starb $^{3}$ ) schrieb Goethe: ,Er(Gott) hat uns einen Mann genommen, dessen Leben wir schon einige Jahre an einem seidenen Faden hängen

1) $\mathrm{KuBmaul}$ nannte die motorische Aphasie — ,ataktische“, wobei die Kranken das Gesprochene verstehen, aber die zum Aussprechen eines Wortes gehörenden Innervationen nicht finden können. Vgl. H. Gutzmann, 4. Aufl. von $\mathrm{Ku}$ Bmauls Störungen der Sprache. Leipzig 1910, S. $320 \mathrm{f}$.

2) Vgl. B. Fraenkel, Des jungen Goethes schwere Krankheit. 1910. Zeitschrift für Tuberkulose, Bd. 15 .

$\left.{ }^{3}\right)$ K. Knetsch, Goethes Ahnen. Lpz. 1908. Tafel VI. 
sahen, dessen feuriger Geist die unterdrückende Last eines kranken Körpers mit schwerer Ängstlichkeit fühlen mußte."

Wegen etwaiger genauerer Angaben über J.W. Textors Krankheiten wandte ich mich an Herrn Prof. Dr. Heuer in Frankfurt a. M., der mir aber nur mitteilte, daß über Krankheiten Textors nichts bekannt sei; er sei bis ins Alter immer gesund gewesen, habe dann vor seinem Tode längere Zeit gekränkelt. Ich stehe aber nicht an, die oben erwähnten präzisen Angaben von H.Düntzer irgendwie in Zweifel ziehen zu sollen, glaube vielmehr mit aller Bestimmtheit, daß Goethe bei der geradezu klassischen Schilderung seinen Großvater Textor vor seinem geistigen Auge gesehen hat.

Was mir an der Goetheschen Stelle die Aphasie bei rechtsseitigem Schlaganfall betreffend, besonders wichtig erscheint, ist, daß Goethe, da er erst 1805, von Lauchstedt kommend, in Halle mit Gall zusammentraf und dessen Kurse besuchte ${ }^{1}$ ), es wohl außer allem Zweifel steht, daß etwa Gallscher Einfluß für diese Stelle heranzuziehen ist.

Aus der von Troussea u gegebenen Entwicklung der Lehre von der Aphasie entnehme ich (a.a. O., S.636) noch, daß Da x bereits 1800 gesehen hatte, daß die mit Aphasie behafteten Kranken, wenn sie paralytisch waren, ihre Paralyse auf der rechten Seite und folglich die Verletzung in der linken Gehirnhemisphäre hatten. Dax erkannte auch bald, daß die Verletzung, sobald das Wortgedächtnis Not litt, stets auf der linken Seite ihren Sitz hatte und fügte hinzu, daß er niemals dieses Vergessen der Wörter in solchen Fällen beobachtet hatte, wo die Erkrankung des Gehirns sich ausschließlich auf die rechte Hemisphäre geworfen hätte.

Selbst ein so ausgesprochener klinischer Beobachter wie van Swieten (1700-1772) kannte zwar, wie aus den folgenden Worten hervorgeht, offenbar die Aphasie in unserem Sinne, aber er war auf dem Wege der Erkenntnis noch nicht so weit vorgeschritten wie Goethe (1797) und $\mathrm{Dax}(1800)$, daß er die Beobachtung machen konnte, daß rechtsseitige Hemiplegie am häufigsten mit Sprachstörungen einhergehen. Van Swieten schreibt 1753 in seinen Kommentarien, an denen er etwa 30 Jahre arbeitete (§ 1018):

„Vidi plures, qui ab apoplexia curati omnibus functionibus cerebri recte valebant, nisi quod deesset hoc unicum, quod non possunt vera rebus designandis vocabula invenire; manibus, pedibus, totius corporis nixu conabantur explicare miseri, quid vellent, nec poterant tamen. Malum illud per plures annos saepe insanabile perstat. " 2 )

Es ist um so auffallender, daß van Swieten an dieser Stelle der

1) Vgl. Magnus, Goethe als Naturforscher. Lpz. 1906, S. 34 u. Froriep, a. a. O., S. 7 f. und vor allem Möbi us, Goethe, Teil 2, S. 209-238.

$\left.{ }^{2}\right)$ van Swieten, Commentaria in H. Boerhaave Aphorismos. Bd. 3. LugJuni Batavorum 1753, p. 288. 
rechtsseitigen Hemiplegie nicht Erwähnung tut, weil er einige Sätze weiter unten von $\mathrm{Mal} \mathrm{pighi}^{1}$ ) (gest. 1694) erzählt, daß dieser bedeutende Forscher, der Stein- und Gichtschmerzen und mannigfaches Unglück großmütig ertragen hatte, ,ab apoplexia, et totius dextri lateris paralysi, curatus, magnam in memoria et ratiocinium laesionem habuit, et quavis minima de causa lacrymabatur."

Goethe hat also 1796, vier Jahre vor Dax, die ausgezeichnete klinische Beobachtung gemacht, daß rechtsseitiger Schlaganfall und Sprachstörungen zusammen in Erscheinung treten ${ }^{2}$ ).

Eine weitere, die Aphasie betreffende Stelle, die Bryk (a.a. O.) unbekannt geblieben ist, findet sich in Wilhelm Meisters Wanderjahre ${ }^{3}$ ), die 1829 erschienen: Es heißt dort (3. Buch, 13. Kapitel:

..., ,mich ereilte noch ein anderes Urteil, daß mein Vater, vom Schlag gerührt, zwar noch sinnliche Kenntnis von der Welt, aber weder geistige noch körperliche Tätigkeit gegen dieselbe behalten hat.... Sie ließ mich in eine ... Stube treten, wo der Alte unbeweglich im Sessel saß. Er hatte sich wenig geändert. Ich ging auf ihn zu, er sah mich erst starr, dann mit lebhafteren Augen an; seine Züge erheiterten sich, er versuchte die Lippen zu bewegen, und als ich die Hand hinreichte, seine ruhende zu fassen, ergriff er die meine von selbst, drückte sie und sprang auf, die Arme gegen mich ausstreckend. ,o Gott!' rief er, ,der Junker Lenardo! er ist's, er ist es selbst!‘ Ich konnte mich nicht enthalten, ihn an mein Herz zu schließen; er sank in den Stuhl zurück, die Tochter eilte hinzu, ihm beizustehen; auch sie rief: ,Er ist's! Sie sind es Lenardo!"

Die jüngere Niohte war herbeigekommen, sie führten den Vater, der auf einmal wieder gehen konnte, der Kammer zu, und gegen mich gewendet, sprach er ganz deutlich: ,Wie glücklich, glücklich! bald sehen wir uns wieder!‘

In dieser zweiten Beobachtung Goethes haben wir es mit einem plötzlichen Aufhören des aphatischen Symptomenkomplexes zu tun, wie er tatsächlich „,zuweilen selbst bei einer kompletten Zerstörung des Brocaschen Gebiets erfolgen kann" [Bastian")]. Da die Forschungen über Aphasie erst um die Zeit, als Goethe diese letzten Bemerkungen schrieb, einsetzten, so verdient auch diese Stelle unsere Beachtung.

Wenn man bei dieser Stelle auch nach einem Vorbild suchen will, so hatte ich daran gedacht, daß Goethe bei dieser Schilderung seinen Vater Johann Caspar Goethe (geb. 1710, gest. 1782) in Erinnerung

1) Vgl. Baglivi, Opera omnia medico-practica. Lugduni Batavorum 1704, p. 621. - Editio C. G. Kühn, Bd. 2. Lpz. 1828, S. 380.

$\left.{ }^{2}\right)$ Vgl. auch Jastrowitz, Berl. klin. Wochenschrift 1875, Nr. 23 und derselbe, Goethe-Jahrbuch XVI (1895). S. $192 \mathrm{f}$.

$\left.{ }^{3}\right)$ Hg. von H. Maync. Bd. 11, ebenda S. 398 f. u. 461.

4) H. Ch. Bastian, Utber Aphasie und andere Sprachstörungen. Lpz. 1902. S. $450 \mathrm{ff}$. u. 459 oben. Auch kann, wie E. Niessl von Ma yendorf (Die aphasischen Symptome usw. Lpz. 1911, S. 25 f.) angibt, bei der Amnesia verbalis kinaesthetica noch nach 6 Monaten die Sprache wiederkehren (de Font Reault). 
vor sich sah, der schon 1776 Schlaganfälle bekam und beim Tode Cornelias krank war; er wurde in den folgenden Jahren still und anteillos; „er war ein gebrochener Mann“. Am 20. September 1779 schrieb der Sohn: ,Meinen Vater habe ich verändert angetroffen, er ist stiller und sein Gedächtnis nimmt ab." 1781 erlitt der Vater einen neuen Insult, von dem er ,körperlich sich notdürftig wiederherstellte, aber er blieb geistesschwach"; ,ein neuer Schlag beraubte ihn aller Bewegung; jetzt war er auch geistesabwesend, bedurfte beständiger Aufsicht, war sich und anderen zur Last" ". Nach längerem Leiden starb er am 25. Mai 1782 ganz unerwartet im 72. Jahre. -

Soweit Düntzer ${ }^{1}$ ). Prof. Dr. Heuer teilt mir gleichzeitig mit, daß sich der Herr Rat J. C. Goethe ebenfalls wie Textor einer vorzüglichen Gesundheit bis etwa 1777 erfreut hat. „Er ist dann an einem Gehirnleiden, wohl Erweichung erkrankt und hat, wie es scheint, zwei Schlaganfälle gehabt."

„Der sehr normale und geistig lebendige Mann, “ fährt He u er²) fort, ,,ist dann zunächst etwas ,wunderlich` geworden, immer ängstlicher (auch in Geldsachen, so daß er, ganz gegen seine frühere Art, geizig und knauserig erschien, sehr weich, durch jede Aufregung zu Tränen gerïhrt und außer Fassung gebracht. Zuletzt habe er, nach Aussage seiner Frau, nur noch ein vegetatives Dasein geführt. Das Leiden muß sich sehr allmählich entwickelt haben, da seine Umgebung es zuerst gar nicht als solches erkannte."

Wenn ich diese Notizen über die Krankheit von Goethes Vater mit der obigen Stelle zusammenhalte, so muß ich allerdings sagen, daß sich auch ein Wahrscheinlichkeitsbeweis für diesen Fall nicht geben läßt, da $B$ aber die erste Stelle wohl unzweifelhaft auf den alten Textor zu beziehen ist.

Jedenfalls zeigt sich $\mathrm{G}$ o e t $\mathrm{h} \mathrm{e}$ in den beiden klinischen Schilderungen des aphatischen Symptomenkomplexes wieder als derjenige, wie Helm holtz ${ }^{3}$ ) sich ausdrückt, ,dessen umfassendes Talent namentlich in der besonnenen Klarheit hervortrat, womit er die Wirklichkeit des Menschen und der Natur in ihren kleinsten Zügen mit lebensfrischer Anschauung festzuhalten und wiederzugeben wußte".

Anmerkung bei der Korrektur: Herr Prof. H. Maync macht mich bei dieser Gelegenheit auf einen ähnlichen Fall von Aphasie aufmerksam. den der Dichter Eduard Mörike bei seinem Vater, der 54 jährig starb, beschreibt. (Mörikes Werke, herausg. von Ma ync, Bibliograph. Institut, Bd. 2, S. 458ff.). Mörike, der bei dessen Tode

1) Vgl. Düntzer, Goethes Stammbäume, Gotha 1894, S. 153 f. u. Goethe von Möbius. Teil l, S. 233.

$\left.{ }^{2}\right)$ Nach He uer geben die neuesten in Betracht kommenden Schriften von R. So $m$ mer, Goethe im Lichte derVererbungstheorie 1908 u.C. Knets $\mathrm{ch}$, Goethes Ahnen (1908) auch nichts Bestimmtes.

3) H. Helmholtz, Über Goethes naturwissenschaftliche Arbeiten (1853) in: Vorträge, Hefと 1 (1865), S. 33. 
13 Jahre alt war, spricht aber von der Lähmung der ganzen linken Seite (statt der rechten) und der beinahe völligen Lähmung der Sprachwerkzeuge. - Viel mehr Beachtung verdienen die Briefstellen, in denen Wilhelm Heinse (1746-1802) seinen im 56. Lebensjahre erlittenen Schlaganfall selbst beschreibt. Unter dem 27. Juni 1802 schreibt Heinse an Sömmering: „wen die Götter lieben, der stirbt jung", und am 29. fügt er diesem Briefe lakonisch die Worte bei: „Gleich dara uf überfiel ihn eine Paralysis. Er konnte nicht sprechen; die Zunge, der rechte Arm und das rechte Bein waren ihm gelähmt". Doch erholte sich Heinse schnell, so daß er am 1. Juli dem Freunde mitteilt: „Ich kann wieder sprechen, gehen, sehr schlecht zwar, doch wieder schreiben..."; am 4. Juli meint er, daß der „Blitz vom Himmel" herab auf seinen Scheitel noch gnädig war; aber fast ein Jahr nach dem ersten trat ein zweiter Insult am 17. Juni 1803 ein, so daß Heinse in seinem Zimmer am Boden liegen blieb. (Vgl. W. Heinse, Briefe, Bd. 2, S. 336ff., herausg. von Carl Schüddekopf, mit einer Einführung. 1913.) Fünf Tage später, am 22. Juni 1803, erlag Heinse diesem letzten Schlaganfall. 